\title{
Preventing chronic disease in people with mental health problems: the HEALTH Passport approach
}

\author{
Naomi Anderson, ${ }^{1}$ Sanjana Sridharan, ${ }^{2}$ Matthew Megson, ${ }^{1}$ Amy Evans, ${ }^{3}$ James Vallance, ${ }^{3}$ \\ Swaran Singh, ${ }^{1}$ Rashmi Shukla, ${ }^{4}$ Vinod Patel ${ }^{1,3}$
}

The Psychiatrist (2012), 36, 208-213, doi: 10.1192/pb.bp.111.036533

${ }^{1}$ Warwick University, Coventry; ${ }^{2}$ Caludon Centre, Coventry; ${ }^{3}$ George Eliot Hospital NHS Trust, Nuneaton; ${ }^{4}$ Department of Health

Correspondence to Vinod Patel (vinod.patel@warwick.ac.uk)

First received 19 Aug 2011, final revision 18 Nov 2011, accepted 13 Dec 2011

\begin{abstract}
Aims and method The HEALTH Passport is a tool to help patients make lifestyle changes to reduce the future burden of chronic disease. This study assesses the potential of this behaviour change strategy in psychiatric patients. We introduced 50 psychiatric in-patients to the HEALTH Passport and asked them to complete a semi-qualitative questionnaire. Results were compared with those of 100 controls.
\end{abstract}

Results Psychiatric in-patients are exposed to almost twice as many modifiable risk factors of chronic disease compared with controls. Although psychiatric in-patients are less motivated to address their risk factors, the HEALTH Passport could almost halve the proportion of psychiatric patients at high risk of chronic disease.

Clinical implications The low level of health literacy among psychiatric patients must be addressed to reduce their risk exposure. Potentially, the HEALTH Passport provides a cost-effective tool for this purpose.

Declaration of interest None.
People with mental health problems can lose over 20 years of potential life compared with the general population. ${ }^{1}$ Although suicides contribute to this figure, the mortality rate due to natural causes such as cardiovascular disease is up to four times that of the general population. ${ }^{2}$

The World Health Organization attributes $80 \%$ of heart disease, strokes and type 2 diabetes mellitus to four modifiable risk factors: smoking, poor diet, physical inactivity and alcohol. ${ }^{3}$ Exposure to these risk factors is known to be significantly higher in people with mental illness, but normal interventions such as nicotine replacement therapy are less effective in this group of people. ${ }^{4}$

The HEALTH (Helping Everyone Achieve Long Term Health) Passport (Fig. 1 and online Fig. DS1) is a patientheld record designed to improve health literacy and motivate patients to make lifestyle changes to improve their long-term health. The HEALTH Passport provides patients with two evidence-based facts and an action plan for each of the ten most important modifiable risk factors of chronic disease. These can be summarised using the following $\mathrm{ABCD}$ approach.

- Advice: do not smoke; maintain a normal body weight; exercise for at least $30 \mathrm{~min}$, five times a week; consume five portions of fruit and vegetables daily; drink responsibly; practise safe sex; do not take illicit drugs; participate in activities that increase emotional wellbeing; attend cancer screening.

- Blood pressure control.

- Cholesterol control.
- Diabetes risk reduction: do not smoke; eat healthily; maintain a normal weight; exercise regularly.

The HEALTH Passport is designed to be used in healthcare consultations using the Comprehensive Health Assessment Talk protocol. This takes 5-10 min to conduct:

1. The patient is introduced to the HEALTH Passport.

2. The patient's health score is assessed.

3. The patient is congratulated on their achievements and advised of the importance of maintaining these achievements.

4. Risk factors are selected for attention/elimination. An action plan is agreed, ensuring that all goals are specific, realistic and achievable.

5. Progress is assessed at the patient's next medical review (iteration of steps $2-5$ ).

The HEALTH Passport is a public domain document. Electronic copies are freely available as an online supplement to this paper.

\section{Method}

Nursing staff working in the general adult psychiatry wards at the Caludon Centre, Coventry, were asked to identify all current in-patients who were well enough to maintain concentration for 10-15 min (the time taken to complete the semi-qualitative study questionnaire). Control participants were recruited from out-patient clinic waiting rooms at 

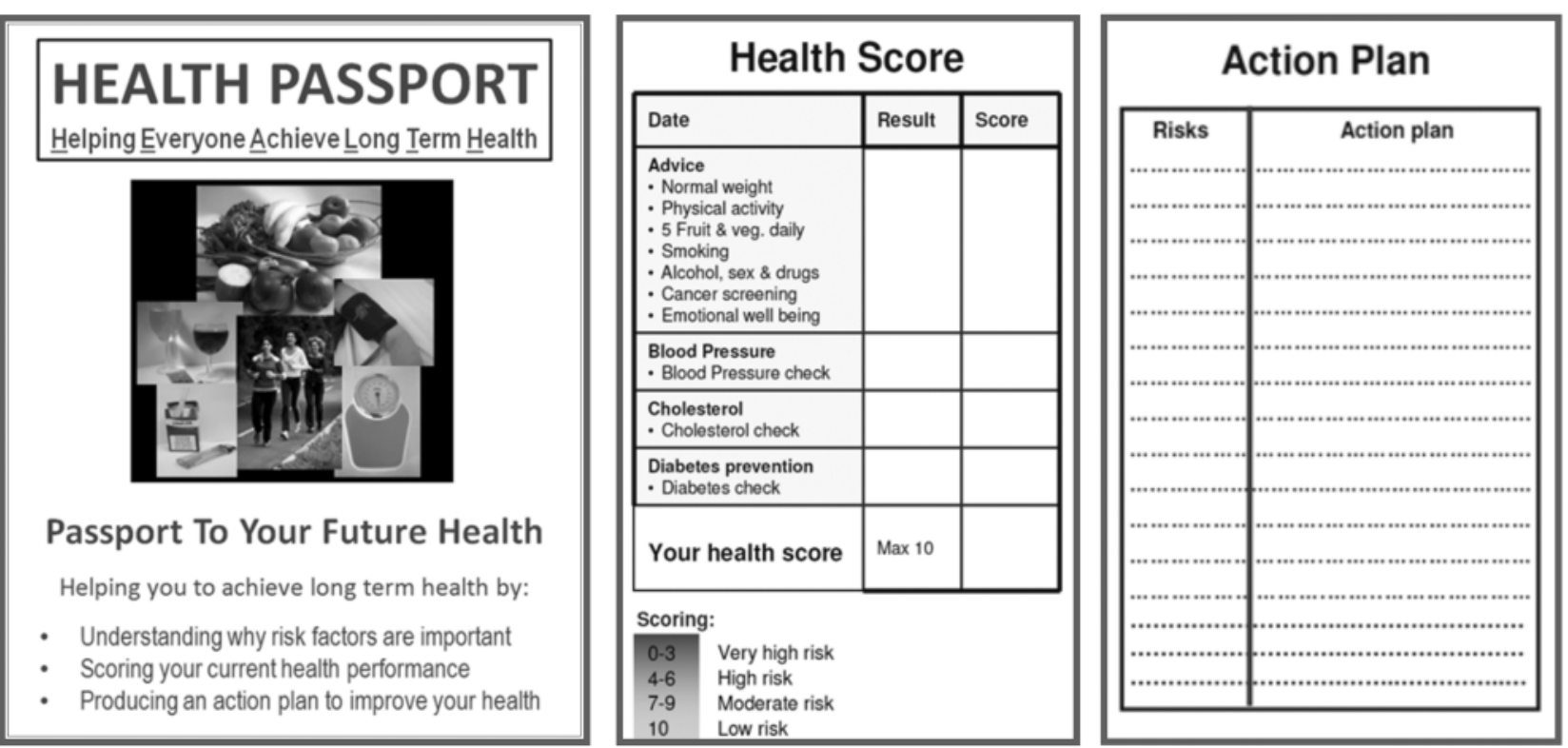

Fig. 1 The HEALTH Passport. The passport contains two evidence-based facts and an action plan for each of the ten risk factors. Patients work with a healthcare professional to calculate their current health score and to develop an individualised action plan. The full passport is available in an online supplement to this paper.

George Eliot hospital, Nuneaton, and from the Muslim Community Centre, Nuneaton.

Participants were briefly introduced to the HEALTH Passport and the objectives and requirements of our research project. Consent was obtained. Participants were asked to complete a tick-list to assess their current exposure to risk factors of chronic disease, and to show which risk factors (if any) they wished to address.

Participants were asked to identify three factors that prevented them living more healthily ('barriers to lifestyle change'). They were also asked to rate the importance of their long-term health on a scale of 1-10 (with 10 representing very important).

To assess the participants' response to the HEALTH Passport, patients were asked how often (never/rarely/ sometimes/often/always) they liked learning about their health and healthcare, whether or not they liked the HEALTH Passport as a method of teaching (yes/no/ unsure), and what new information they had learnt from the HEALTH Passport (to assess their existing level of health literacy). They were asked what support they would want or need to use the HEALTH Passport, and whether they would prefer to work through the passport individually or in groups. Finally, they were asked to identify where they obtained health-related information.

\section{Definitions}

The current risk exposure is defined as the number of risk factors that a participant is currently exposed to (scoring one point for each of the ten risk factors). Yusuf and colleagues demonstrated the cumulative adverse effect of exposure to multiple risk factors. ${ }^{5}$ Based on this, participants exposed to four or more risk factors are considered to be at high risk of developing chronic diseases.
The relative risk exposure is a measure used to compare the risk exposure in the psychiatric group with the control group. It is defined as: relative risk= proportion of psychiatric group exposed/proportion of control group exposed.

Patients were asked which risk factors they would like to focus on eliminating. For each risk factor, the aspired improvement ratio is defined as the proportion of at risk patients expressing a desire to change. Thus, aspired improvement ratio=number of participants who will address the risk factor/number of participants currently exposed to the risk factor.

\section{Statistical analysis}

Responses were analysed using Excel 2007 and the statistical package R version 2.14.0 (The R Foundation for Statistical Computing, Vienna, Austria; www.r-project.org). The results were compared with results from the control group. The $\chi^{2}$ test, the Wilcoxon rank sum test, Student's $t$-test and Fisher's exact test were used to calculate $P$-values. As the sample sizes were small, Yates' continuity correction factor was used for $\chi^{2}$ calculations to avoid underestimating $P$-values. Results were considered significant if $P<0.05$.

\section{Results}

Over 6 weeks, 53 psychiatric in-patients consented to take part in the study, equating to approximately $50 \%$ of the in-patient admissions (80\% of eligible in-patients) in general adult psychiatry wards within that time. Of the patients interviewed, 3 withdrew before sufficient information had been recorded, leaving a sample of 50 patients. Although no age restrictions were formally imposed, the sample did not include patients from the old age psychiatric wards, all patients were aged over 16 years, and patients in 
the psychiatric intensive care unit were excluded from the study.

The psychiatric sample comprised 50 participants (31 males, 19 females), with a mean (s.d.) age of 40.9 (13.6) years. Of these, $26(52 \%)$ volunteered their diagnoses: physical conditions $(n=8,31 \%)$, depression $(n=4,22 \%)$, bipolar affective disorder $(n=4,22 \%)$, personality disorder $(n=2$, $11 \%)$, schizophrenia $(n=2,11 \%)$, eating disorder $(n=2,11 \%)$, anxiety disorder $(n=1,6 \%)$ and 'mental illness' $(n=3,17 \%)$.

Regarding controls, 154 individuals were introduced to the study. Overall, 20 participants declined to participate, 9 participants were unable to participate because they did not have their reading glasses with them, and 22 participants were required to attend their appointment before they completed the questionnaire. One participant withdrew before completing the questionnaire, and a further two participants completed the questionnaire incorrectly and were excluded from the analysis. All participants were lay individuals and not healthcare professionals.

The final control sample comprised 100 participants (22 males, 78 females), with a mean (s.d.) age of 48.7 (16.1) years. Out-patients were attending antenatal $(n=1)$, audiology $(n=3)$, breast $(n=2)$, diabetes $(n=12)$, endocrinology $(n=9)$, endoscopy $(n=1)$, ear-nose-throat $(n=4)$, eye $(n=2)$, fracture $(n=1)$, gastrointestinal $(n=1)$, general medicine $(n=1)$, chronic fatigue syndrome $(n=1)$, orthopaedic $(n=3)$, renal $(n=1)$, respiratory $(n=2)$, rheumatology $(n=1)$, surgery $(n=7)$ and urology $(n=1)$ clinics. Twenty-five participants were accompanying patients to their appointments and seventeen participants were staff members. A further five participants were from the Muslim Community Centre.

Age- and gender-specific variations in every parameter were assessed within each group and were found not to be statistically significant.

\section{Current risk exposure}

As shown in Fig. 2, the current risk exposure was significantly higher in the psychiatric group $(P<0.001$; Wilcoxon rank sum test), with a median of 5.0 (2.5th and 95th centiles 2.0-8.8), compared with 2.5 (2.5th and 95th centiles 0.0-7.0) in the control group. Furthermore, 78\% (95\% CI 76.4-79.6) of the psychiatric group were at high risk of developing chronic disease, compared with $37 \%$ (95\% CI 36.1-37.9) of controls. This difference is significant at the 99.9\% confidence level $\left(P<0.001, \chi^{2}=20.81\right)$.

\section{Exposure to individual risk factors}

Figure 3 illustrates participants' exposure to individual risk factors. The relative risk exposure was significantly greater than unity $\left(P<0.05\right.$ using the $\chi^{2}$ test with Yates' continuity correction) for the following risk factors: 4.2 (95\% CI 2.357.33) times as many psychiatric participants $(54 \%, 95 \%$ CI 52-56) as controls (13\%, 95\% CI 12-14) smoked, and 2.8 (95\% CI 1.34-5.85) times as many psychiatric participants (28\%, 95\% CI 26-30) as controls (10\%, 95\% CI 9.4-11) misused alcohol and drugs or practised unsafe sex.

In addition, 2.6 (95\% CI 1.62-4.18) times as many psychiatric participants (52\%, 95\% CI 50-54) as controls (20\%, 95\% CI 19-21) stated they lacked emotional wellbeing, and 2.0 (95\% CI 1.50-2.54) times as many psychiatric participants $(82 \%, 95 \%$ CI $80-84)$ as controls $(42 \%$, 95\% CI 41-43) were at high risk of developing diabetes as a result of lifestyle choices.

\section{Potential risk reduction}

The aspired improvement ratios were significantly higher in the control group than the psychiatric group for almost all risk factors (Fig. 4). Despite this, if psychiatric participants could achieve their aspired lifestyle changes, their total risk exposure would be reduced by an average of 1.7 risk factors per person, sufficient to reduce the number of psychiatric participants at high risk of chronic disease from $78 \%$ to $46 \%$ (a $41 \%$ reduction).

Of the ten risk factors, psychiatric participants demonstrated greatest motivation to exercise more, to reduce their alcohol and drug consumption, and to monitor their blood pressure. If the participants' aspired lifestyle

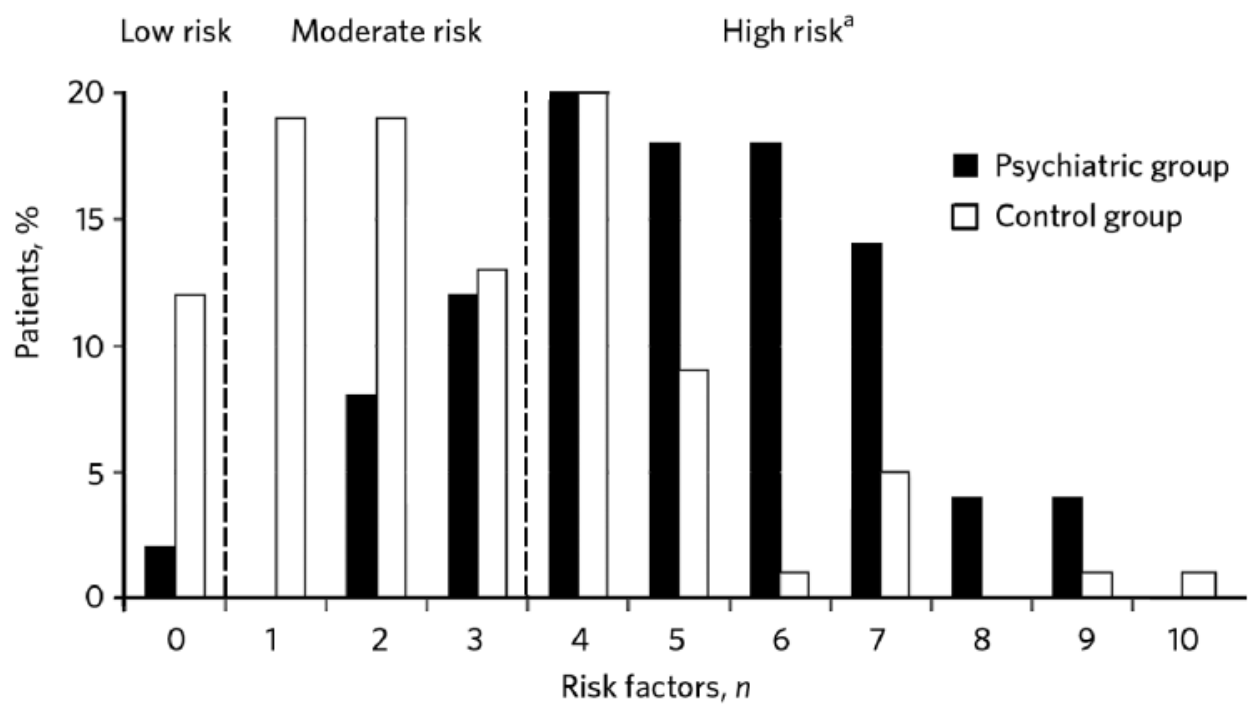

Fig. 2 Number of modifiable risk factors to which participants are currently exposed. Individuals exposed to four or more risk factors are at high risk of developing chronic disease. a. Psychiatric group, 78\%; control group, $37 \%$. 


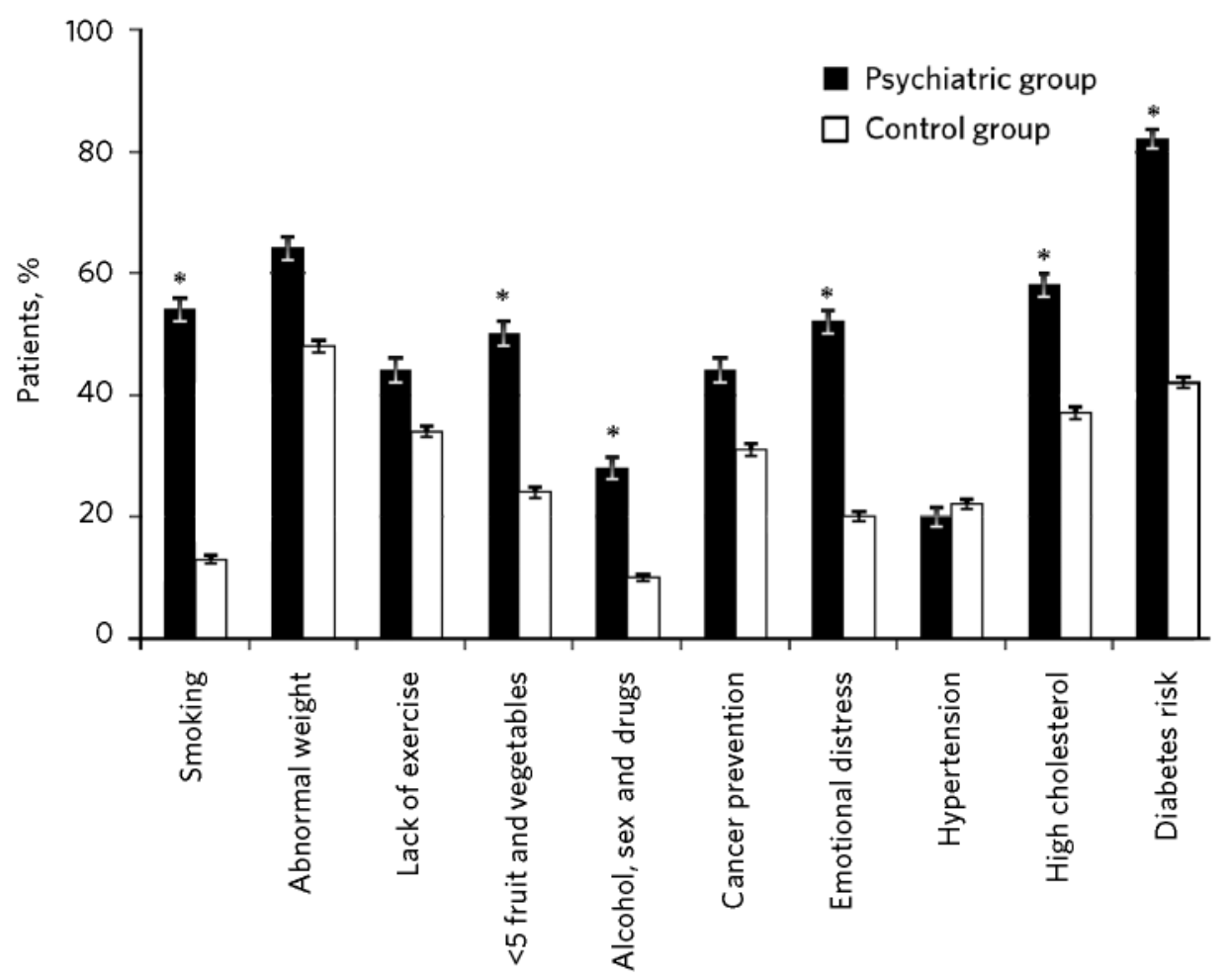

Fig. 3 Current exposure to individual risk factors among psychiatric and control groups. Error bars indicate the $95 \%$ confidence interval for each proportion. Asterisks $\left(^{\star}\right)$ indicate risk factors for which the relative risk $(R R)$ exposure is greater than unity $(R R>1)$ with $P<0.05$.

changes could be translated into actions, the incidence of physical inactivity among psychiatric participants could be reduced by $55 \%$ (95\% CI 50-59), and misuse of alcohol and drugs could be reduced by $50 \%$ (95\% CI $43-57)$. Use of the HEALTH Passport could reduce the incidence of obesity by up to $44 \%$ (95\% CI 41-47) and the incidence of smoking by up to $37 \%$ (95\% CI $34-41)$.

\section{Barriers to lifestyle change}

The most important barriers to lifestyle change among the psychiatric group were enjoyment of unhealthy behaviours (42\%), lack of motivation (36\%) and lack of energy (24\%). By comparison, the most important factors among the control group were lack of time (36\%), lack of energy (20\%) and pain (19\%).

When asked to rate the importance of their long-term health, the rating was significantly lower in the psychiatric group $(P<0.001$, Wilcoxon rank sum test), with a median of 9 (2.5th and 95th centiles 1-10), compared with 10 (2.5th and 95th centiles 6.5-10) in the control group. Significantly fewer psychiatric participants $(48 \%)$ than controls $(70 \%)$ rated the importance of their long-term health as ' $10 / 10$ ' $\left(\chi^{2}=5.99\right.$, d.f. $\left.=1, P=0.014\right)$.

\section{Response to the HEALTH Passport}

Of the psychiatric participants, 86\% (95\% CI 85-87) stated they enjoyed learning about healthcare at least sometimes. Further, 92\% (95\% CI 91-93) said they liked the HEALTH Passport method of teaching; 96\% (95\% CI 95-97) wanted to work through the HEALTH Passport with a healthcare worker rather than on their own; and $76 \%$ (95\% CI 74-78) wanted individual rather than group coaching.

Compared with only $25 \%$ of controls, $60 \%$ of psychiatric participants stated they learnt new information from the HEALTH Passport, indicating a significantly lower level of health literacy in the psychiatric group. The risk factors about which most psychiatric participants learnt new information were weight control $(32 \%)$, diet (32\%), cholesterol (30\%), mental well-being (28\%) and diabetes (28\%). By comparison, $16 \%$ of controls learnt new information about diabetes, and $13 \%$ learnt more about cholesterol and cancer screening.

\section{Sources of health-related information}

In the control group, $57 \%$ of participants identified using the internet to obtain health-related information (most popular), $44 \%$ identified turning to doctors, and $30 \%$ identified using television. By contrast, in the psychiatry group, $66 \%$ participants identified using doctors to obtain health-related information (most popular), 38\% identified using television, and $34 \%$ identified the internet.

\section{Discussion}

In line with the literature, ${ }^{1,2,4,6}$ our results demonstrate that people with severe mental health problems are at increased risk of physical comorbidity and mortality. They are exposed to a greater number of modifiable risk factors for chronic disease and are less motivated to address these risk factors.

In our study sample, over four times as many psychiatric participants as controls smoke, almost three 


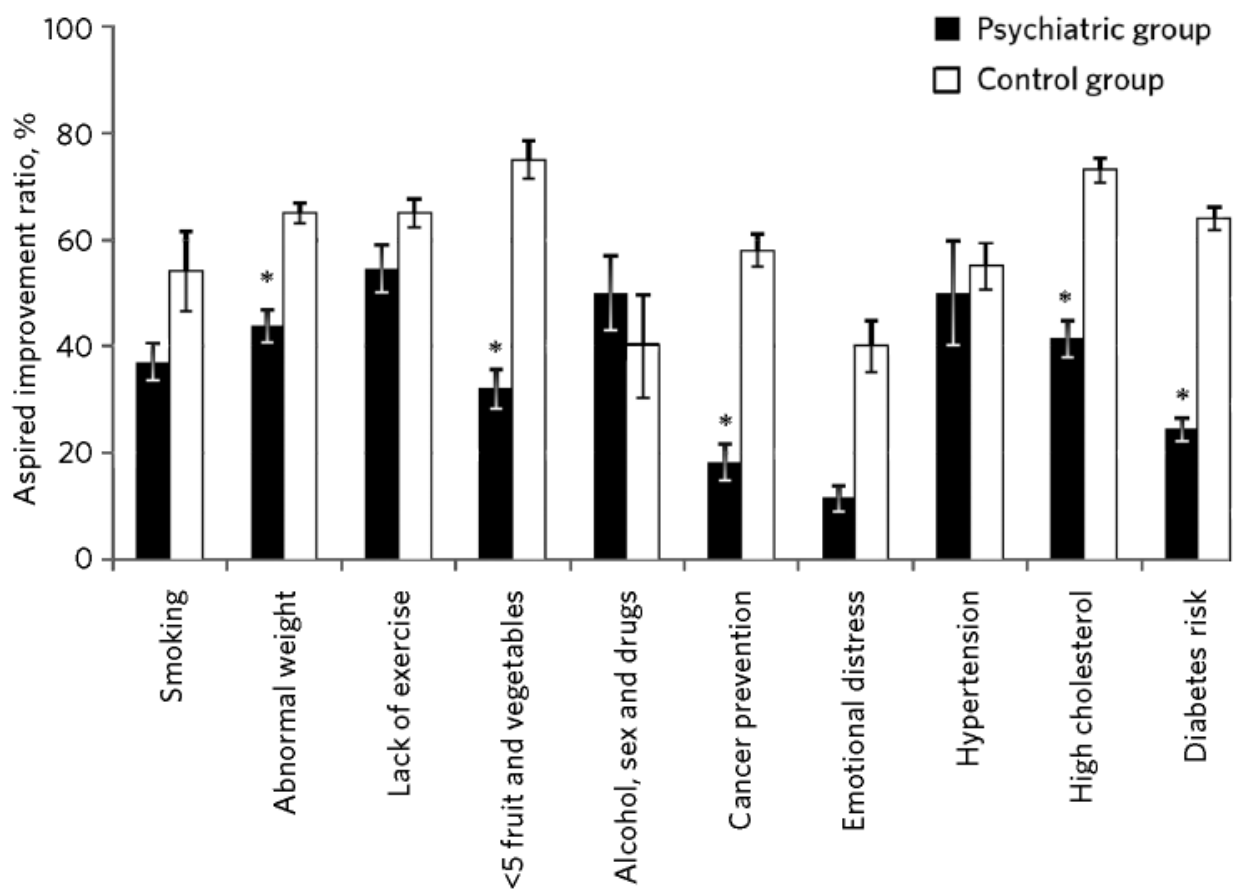

Fig. 4 Motivation among psychiatric and control groups to address individual risk factors. The aspired improvement ratio (AIR) is defined as the proportion of at-risk individuals expressing a desire to change. The error bars indicate the $95 \%$ confidence intervals for aspired improvement ratios. Asterisks $\left({ }^{\star}\right)$ indicate risk factors for which the relative improvement in the psychiatric group is significantly less than that of the control group $\left(A I R_{\text {Psych }} / A I R_{\text {Control }}<1\right)$, valid at the $95 \%$ confidence level $(P<0.05)$.

times as many misuse alcohol and drugs, and twice as many are at risk of developing diabetes as a result of lifestyle choices (before the diabetogenic effect of psychiatric medications and non-modifiable risk factors are considered). These risk ratios are in line with figures published by De Hert and colleagues for patients with schizophrenia. ${ }^{7,8}$

Of the psychiatric participants, $78 \%$ were exposed to four or more modifiable risk factors of chronic disease almost twice as many psychiatric patients as controls were in the high-risk category. The psychiatric participants' motivation to address individual risk factors (calculated as the proportion of at-risk participants expressing a desire to eliminate that factor) was significantly lower than that of the controls for almost all risk factors. For example, only $24 \%$ of at-risk psychiatric participants wanted to reduce their risk of developing diabetes, compared with $64 \%$ of at-risk participants in the control group.

Despite this, there is significant scope for improvement. Psychiatric participants identified, on average, 1.7 risk factors that they were keen to address. Therefore it is important to support patients in making whatever changes they feel ready and able to make.

\section{Clinical application of the HEALTH Passport}

The HEALTH Passport is a behaviour change strategy. It provides a simple way for health professionals to assess how best to help their patients address their risk factors. For example, an individual who smokes, is overweight and is physically inactive may not feel able to stop smoking or to address their weight, but committing to $20 \mathrm{~min}$ of exercise once a week is a good start.
Choice is empowering, so it is paramount that the patient is enabled to make informed decisions and to set their own goals. Care must be taken, however, to ensure that the individual's agreed action plan is realistic, timeoriented, specific and achievable. By supporting people to achieve these goals, we can help them develop greater selfefficacy and hence help them to increase their perceived behavioural control. In turn, this enables individuals to build on their successes. Goals may be modified or extended during follow-up, and patients should be encouraged to talk about their difficulties. Relapses are to be expected and must be approached constructively.

Over time, promoting such lifestyle changes (however small the steps appear) will help reduce the burden of chronic disease on society. If psychiatric patients can be helped to achieve their aspired lifestyle changes, then this could approximately halve the number of patients falling into the high risk of chronic disease category, with a $50 \%$ reduction in physical inactivity and misuse of alcohol and drugs.

\section{Health promotion within psychiatric institutions}

Physical activity was the risk factor that patients felt most able and motivated to address. A number of psychiatric patients, however, particularly young men, commented on the lack of a gym or other exercise facilities in hospital. Considering the evidence that physical activity (particularly participation in sports) can be beneficial to both mental and physical health, ${ }^{9,10}$ healthcare providers must seriously consider providing exercise facilities for psychiatric in-patients. 
Of the psychiatric patients in our study, $66 \%$ stated they relied on doctors and other healthcare professionals for health education. In view of this, psychiatric hospitals must strive to educate by example. For instance, unless we provide healthy hospital meals with a high fruit and vegetable content, we cannot expect patients to eat healthily at home. Patients commented that large carbohydrate-rich hospital meals made them put on weight, providing a barrier to lifestyle changes. As diet, weight control, cholesterol and diabetes prevention are all areas that patients felt underinformed about, the use of posters in psychiatric hospital canteens could help to improve patients' level of health literacy.

\section{Limitations of the study}

This study looks at patients' current risk exposure and their motivation to reduce their exposure to the modifiable risk factors of chronic disease. It is not possible to elaborate how patients' expressed desires differ from their motivation to change, or to predict the proportion of patients who will succeed in attaining their expressed desires. A full randomised controlled trial is needed to quantitatively assess the efficacy of the HEALTH Passport in reducing the incidence of chronic disease in psychiatric patients.

\section{Implications}

In 2008 mental illness was estimated to cost England around $£ 77$ billion per year. ${ }^{9}$ A significant proportion of this cost is associated with treating patients' physical comorbidities. In the current financial climate, costeffective interventions to reduce the economic burden of disease are highly sought after. No formal cost-benefit analysis has been performed for the HEALTH Passport, but it is highly probable that it is a cost-effective tool to reduce patients' risk of developing chronic disease in the future.

The HEALTH Passport covers all the main modifiable risk factors identified by the National Institute for Health and Clinical Excellence for development of long-term conditions such as diabetes, coronary heart disease, stroke, chronic obstructive pulmonary disease and even common cancers such as lung and colon cancer. ${ }^{11-15}$ This simple passport can become the focus for discussion of risk factors as part of the 'Making Every Contact Count', which is a UK-wide National Health Service initiative to persuade all healthcare professionals to make the most of opportunities (e.g. clinical encounters, waiting rooms) to help people stay healthy and reduce system-wide costs of long-term conditions and certain cancers. ${ }^{16}$

\section{Acknowledgements}

We thank Amitha Gopinath (research assistant, George Eliot Hospital) for the design and layout of the HEALTH Passport, and for conducting the initial literature searches. Further literature reviews and creation of the HEALTH acronym were the work of Steven Laird and Sarah Kinsman (medical students, Warwick Medical School). Thanks also to Matthew Vernon (research fellow, Warwick University School of Life Sciences) for assistance and support with our statistics. The idea of the HEALTH Passport was originally conceived by V.P.

\section{About the authors}

Naomi Anderson is a research assistant and medical student at Warwick Medical School. Sanjana Sridharan is Consultant Psychiatrist at the Caludon Centre, Coventry. Matthew Megson is a medical student at Warwick Medical School. Amy Evans is a research assistant at George Eliot Hospital NHS Trust, Nuneaton. James Vallance is a research assistant at George Eliot Hospital NHS Trust, Nuneaton. Swaran Singh is Head of Division of Mental Health and Wellbeing at Warwick Medical School. Rashmi Shukla is Regional Director of Public Health West Midlands, Department of Health. Vinod Patel is Associate Professor of Clinical Skills at Warwick Medical School; Honorary Clinical Engagement Lead for Long Term Conditions at the NHS West Midlands Strategic Heath Authority Diabetes Centre; and Honorary Consultant in Endocrinology and Diabetes at George Eliot Hospital NHS Trust, Nuneaton.

\section{References}

1 Vreeland B. Bridging the gap between mental and physical health: a multidisciplinary approach. J Clin Psychiatry 2007; 68 (suppl 4): 26-33.

2 Harris EC, Barraclough B. Excess mortality of mental disorder. $\mathrm{Br}$ Psychiatry 1998; 173: 11-53.

3 World Health Organization. 2008-2013 Action Plan for the Global Strategy for the Prevention and Control of Non-Communicable Diseases. WHO, 2008.

4 Tsoi DT, Porwal M, Webster AC. Interventions for smoking cessation and reduction in individuals with schizophrenia. Cochrane Database Syst Rev 2010; 6: CD007253.

5 Yusuf S, Hawken S, Ounpuu S, Dans T, Avezum A, Lanas F, et al. Effect of potentially modifiable risk factors associated with myocardial infarction in 52 countries (the INTERHEART study): case-control study. Lancet 2004; 364: 937-52.

6 Vinogradova Y, Coupland C, Hippisley-Cox J, Whyte S, Penny C. Effects of severe mental illness on survival of people with diabetes. $\mathrm{Br} J$ Psychiatry 2010; 197: 272-7.

7 De Hert $M$, van Winkel $R$, Van Eyck D, Hanssens L, Wampers $M$ Scheen $A$, et al. Prevalence of diabetes, metabolic syndrome and metabolic abnormalities in schizophrenia over the course of the illness: a cross-sectional study. Clin Pract Epidemiol Ment Health 2006; 2: 14.

8 De Hert M, Mauri M, Shaw K, Wetterling T, Doble A, Gindicelli A, et al. The METEOR study of diabetes and other metabolic disorders in patients with schizophrenia treated with antipsychotic drugs. I: methodology. Int Methods Psychiatr Res 2010; 19: 195-210.

9 Government Office for Science. Foresight Mental Capital and Wellbeing Project: Final Project Report - Executive Summary. Government Office for Science, 2008.

10 Asztalos M, Wijndaele K, De Bourdeaudhuij I, Philippaerts R, Matton L, Duvrigneaud $\mathrm{N}$, et al. Specific associations between types of physical activity and components of mental health. J Sci Med Sport 2009; 12: 468-74.

11 National Institute for Health and Clinical Excellence. Brief Interventions and Referral for Smoking Cessation in Primary Care and Other Settings (PH1). NICE, 2006.

12 National Institute for Health and Clinical Excellence. Four Commonly Used Methods to Increase Physical Activity: Brief Interventions in Primary Care, Exercise Referral Schemes, Pedometers and Community-based Exercise Programmes for Walking and Cycling (PH2). NICE, 2006.

13 National Institute for Health and Clinical Excellence. Occupational Therapy Interventions and Physical Activity Interventions to Promote the Mental Wellbeing of Older People in Primary Care and Residential Care (PH16). NICE, 2008

14 National Institute for Health and Clinical Excellence. Alcohol-use Disorders: Preventing the Development of Hazardous and Harmful Drinking (PH24). NICE, 2010

15 National Institute for Health and Clinical Excellence. Prevention of Cardiovascular Disease at Population Level. NICE, 2010.

16 NHS Future Forum. Making Every Contact Count. Department of Health, 2011. 